\title{
Observer-Based Controller Design for a Class of Nonlinear Networked Control Systems with Random Time-Delays Modeled by Markov Chains
}

\author{
Yanfeng Wang, Peiliang Wang, Zuxin Li, and Huiying Chen \\ School of Engineering, Huzhou University, Huzhou, Zhejiang 313000, China \\ Correspondence should be addressed to Yanfeng Wang; neu2009wyf@163.com
}

Received 3 January 2017; Revised 14 February 2017; Accepted 1 March 2017; Published 30 March 2017

Academic Editor: Lei Zou

Copyright (C) 2017 Yanfeng Wang et al. This is an open access article distributed under the Creative Commons Attribution License, which permits unrestricted use, distribution, and reproduction in any medium, provided the original work is properly cited.

This paper investigates the observer-based controller design problem for a class of nonlinear networked control systems with random time-delays. The nonlinearity is assumed to satisfy a global Lipschitz condition and two dependent Markov chains are employed to describe the time-delay from sensor to controller (S-C delay) and the time-delay from controller to actuator (C-A delay), respectively. The transition probabilities of S-C delay and C-A delay are both assumed to be partly inaccessible. Sufficient conditions on the stochastic stability for the closed-loop systems are obtained by constructing proper Lyapunov functional. The methods of calculating the controller and the observer gain matrix are also given. Two numerical examples are used to illustrate the effectiveness of the proposed method.

\section{Introduction}

Networked control systems (NCSs) are spatially distributed systems where the communication between sensor, controller, and actuator is carried out by a shared band limited digital network $[1,2]$. NCSs are used in a wide range of areas such as robots, industrial manufacturing plants, and remote surgery due to their advantages in practical applications, for example, flexible architectures, the reduced weight, simple installation, and maintenance as well as high flexibility and reliability [3,4]. However, the communication networks also present some constraints such as time-delays and packet dropouts result from the limited bandwidth. It is generally known that the time-delay maybe degrades the performance or even causes instability $[5,6]$.

The Markov chain which is a discrete-time stochastic process with the Markov property can be effectively used to model the time-delay in NCSs. The random time-delays in NCSs modeled as Markov chains have been researched in the past several years, and many results have been reported [717]. In [7], the time-delay of NCSs was modeled as a Markov chain, and further a LQG optimal controller design method was proposed. In [8], the NCSs were molded as Markov jump linear systems (MJLSs) where the S-C delay was molded as a finite state Markov chain, and a V-K iteration method was proposed to get a stabilizing controller. In [9], a buffer was added ahead of the actuator, and the time-delays from sensor to actuator were lumped together which was molded as a Markov chain, and then the mean-square stability of the closed-loop system was derived. In [10-12], for the NCSs with S-C delay, the problem of $H_{\infty}$ control was investigated using the Bounded Real Lemma and the Markov jump theory. In $[13,14]$, the $S-C$ delay and C-A delay were modeled as two independent Markov chains. The resulting closed-loop systems were transformed to control systems which contain two Markov chains. The sufficient and necessary conditions for the stochastic stability of the resulting closed-loop systems were established, and the mode-dependent state feedback and output feedback controller were designed, respectively.

The transition probabilities of time-delays in [7-14] were assumed to be completely accessible. However, in practical applications, this assumption is too ideal and hence will limit 


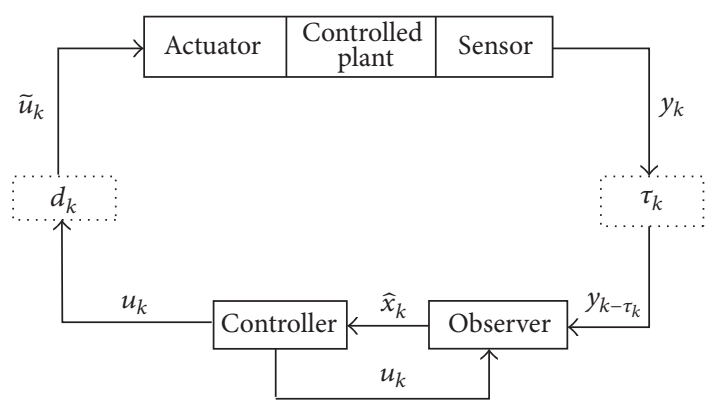

FIgURE 1: Diagram of the NCSs.

the application of the derived results due to the difficulty in obtaining all the transition probabilities of time-delay precisely. Some results have been obtained when the transition probabilities of the time-delays (data packet dropout) are partly inaccessible. In [15], the $H_{\infty}$ control problem was investigated for the NCSs with random data packet dropouts. The closed-loop systems were modeled as MJLSs with four modes and partly inaccessible transition probabilities. In [16, 17], the closed-loop systems were modeled as MJLSs with partly inaccessible transition probabilities of the S-C delay, and the stabilization controller was designed though the linear matrix inequality (LMI) method. In [18], the transition probabilities of the time-delay were assumed to be partly inaccessible, and the fault-tolerant controller for the discretetime NCSs was designed. Unfortunately, in [16-18], only the S-C delay was considered and an improved controller should take both S-C delay and C-A delay into consideration.

It is well known that nonlinearities usually exist in practical systems. Hence, research about nonlinear NCSs is important in both application and theory. To the best of the authors' knowledge, up to now, involving both S-C delay and C-A delay to design the controller for nonlinear NCSs when transition probabilities of S-C delay and C-A delay are both partly inaccessible has not been investigated, which motivates our investigation.

In this paper, we propose two controller design methods for a kind of nonlinear NCSs with both S-C delay and C-A delay based on observer. Compared to the previous relevant works, the main contribution of this paper is that the proposed methods can deal with the situations of both complete accessible transition probabilities and partly inaccessible transition probabilities. The rest of this paper is organized as follows. The closed-loop system model with Markov delays is obtained in Section 2. The main results and proofs are given in Section 3. Section 4 presents the simulation results, and the conclusions are provided in Section 5.

\section{Problem Formulation}

The configuration of the NCSs considering time-delays is depicted in Figure 1 where $\tau_{k}$ and $d_{k}$ denote the S-C delay and C-A delay, respectively.

In this paper, $\tau_{k}$ and $d_{k}$ are modeled as two homogeneous Markov chains which take value in the set $\Omega=\left\{\tau_{m}, \ldots, \tau_{M}\right\}$ and $\Gamma=\left\{d_{m}, \ldots, d_{M}\right\}$, and their transition probability matrices are $\Xi=\left[\lambda_{i j}\right]$ and $\Pi=\left[\pi_{r s}\right]$, respectively. That is, $\tau_{k}$ and $d_{k}$ jump from mode $i$ to $j$ and from $r$ to $s$, respectively, with probabilities $\lambda_{i j}$ and $\pi_{r s}$, which are defined by $\lambda_{i j}=$ $\operatorname{Pr}\left(\tau_{k+1}=j \mid \tau_{k}=i\right), \pi_{r s}=\operatorname{Pr}\left(d_{k+1}=s \mid d_{k}=r\right)$, where $\lambda_{i j} \geq 0, \pi_{r s} \geq 0$, and $\sum_{j=\tau_{m}}^{\tau_{M}} \lambda_{i j}=1, \sum_{s=d_{m}}^{d_{M}} \pi_{r s}=1$, for all $i, j \in \Omega$ and $r, s \in \Gamma$.

In this paper, the transition probabilities of $\tau_{k}$ and $d_{k}$ are both considered to be partly accessible; that is, some elements in matrix $\Xi$ and $\Pi$ are unknown. For notational clarity, $\forall i \in$ $\Omega$, we denote $\Omega=\Omega_{k}^{i}+\Omega_{u k}^{i}$ with $\Omega_{k}^{i}=\left\{j: \lambda_{i j}\right.$ is known $\}$, $\Omega_{u k}^{i}=\left\{j: \lambda_{i j}\right.$ is unknown $\}$. Moreover, if $\Omega_{k}^{i} \neq \varnothing$, it is further described as $\Omega_{k}^{i}=\left\{\Omega_{k_{1}^{i}}, \Omega_{k_{2}^{i}}, \ldots, \Omega_{k_{\mu}^{i}}\right\}(1 \leq \mu \leq e)$, and $\Omega_{u k}^{i}$ is described as $\Omega_{u k}^{i}=\left\{\Omega_{\bar{k}_{1}^{i}}, \Omega_{\bar{k}_{2}^{i}}, \ldots, \Omega_{\bar{k}_{e-\mu}^{i}}\right\}$, where $e$ is the number of elements in the set $\Omega$.

Similarly, $\forall r \in \Gamma$, we denote $\Gamma=\Gamma_{k}^{r}+\Gamma_{u k}^{r}$ with $\Gamma_{k}^{r}=\{s$ : $\pi_{r s}$ is known $\}, \Gamma_{u k}^{r}=\left\{s: \pi_{r s}\right.$ is unknown $\}$. If $\Gamma_{k}^{r} \neq \varnothing$, it is further described as $\Gamma_{k}^{r}=\left\{\Gamma_{k_{1}^{r}}, \Gamma_{k_{2}^{r}}, \ldots, \Gamma_{k_{\theta}^{r}}\right\}(1 \leq \theta \leq \phi)$ and $\Gamma_{u k}^{r}$ is described as $\Gamma_{u k}^{r}=\left\{\Gamma_{\bar{k}_{1}^{r}}^{r}, \Gamma_{\bar{k}_{2}^{r}}, \ldots, \Gamma_{\bar{k}_{\phi-\theta}^{r}}\right\}$, where $\phi$ is the number of elements in the set $\Gamma$.

Considering the following controlled plant after sampling,

$$
\begin{aligned}
x_{k+1} & =A x_{k}+f\left(k, x_{k}\right)+B \tilde{u}_{k}, \\
y_{k} & =C x_{k},
\end{aligned}
$$

where $x_{k} \in R^{n}$ is the state vector, $\widetilde{u}_{k} \in R^{m}$ is the control input, and $y_{k} \in R^{p}$ is the measured output. $A, B$, and $C$ are known real constant matrices with appropriate dimensions. $f\left(k, x_{k}\right)$ is a nonlinear vector function which satisfies the following global Lipschitz conditions:

$$
\begin{aligned}
\left\|f\left(k, x_{k}\right)\right\| & \leq\left\|g x_{k}\right\|, \\
\left\|f\left(k, x_{k}\right)-f\left(k, z_{k}\right)\right\| & \leq\left\|g\left(x_{k}-z_{k}\right)\right\|,
\end{aligned}
$$

where $g$ is a known real scalar.

The dynamic observer-based control scheme is given by

$$
\begin{aligned}
& \text { Observer: }\left\{\begin{array}{l}
\widehat{x}_{k+1}=A \widehat{x}_{k}+f\left(k, \widehat{x}_{k}\right)+B u_{k}+L\left(y_{k-\tau_{k}}-\widehat{y}_{k-\tau_{k}}\right), \\
\widehat{y}_{k}=C \widehat{x}_{k},
\end{array}\right. \\
& \text { Controller: }\left\{\begin{array}{l}
u_{k}=K \widehat{x}_{k} \\
\tilde{u}_{k}=u_{k-d_{k}},
\end{array}\right.
\end{aligned}
$$

where $\widehat{x}_{k} \in R^{n}, u_{k} \in R^{m}$, and $\widehat{y}_{k} \in R^{p}$ are the state vector, control input, and output vector of the observer, respectively. $K \in R^{m \times n}$ is the controller gain and $L \in R^{n \times p}$ is the observer gain.

Remark 1. It should be pointed out that the control input $\tilde{u}_{k}$ of the controlled plant (1) is different from the control input $u_{k}$ of the observer (3) due to the existence of the C-A delay $d_{k}$, while, in the most of the observer-based controller design problem, $\tilde{u}_{k}$ and $u_{k}$ were assumed to be identical.

Define the state estimate errors as

$$
e_{k}=x_{k}-\widehat{x}_{k}
$$


Substituting (3) and (4) into (1) and (5), the closed-loop system can be obtained as follows:

$$
\begin{aligned}
x_{k+1}= & A x_{k}+f\left(k, x_{k}\right)+B K\left(x_{k-\tau_{k}}-e_{k-\tau_{k}}\right), \\
e_{k+1}= & (A+B K) e_{k}+F_{k}-B K x_{k}+B K\left(x_{k-d_{k}}-e_{k-d_{k}}\right) \\
& -L C e_{k-\tau_{k}},
\end{aligned}
$$

where $F_{k}=f\left(k, x_{k}\right)-f\left(k, \widehat{x}_{k}\right)$.

By defining $\eta_{k}^{T}=\left[\begin{array}{ll}x_{k}^{T} & e_{k}^{T}\end{array}\right]^{T}$, the closed-loop system (6) can be written in a compact form as follows:

$$
\begin{aligned}
\eta_{k+1}= & \left(\bar{A}-B_{1} K I_{1}\right) \eta_{k}+B_{2} K I_{1} \eta_{k-d_{k}}-I_{2} L \bar{C} \eta_{k-\tau_{k}} \\
& +\bar{F}_{k},
\end{aligned}
$$

where

$$
\begin{aligned}
\bar{A} & =\left[\begin{array}{ll}
A & 0 \\
0 & A
\end{array}\right], \\
B_{1} & =\left[\begin{array}{l}
0 \\
B
\end{array}\right], \\
B_{2} & =\left[\begin{array}{l}
B \\
B
\end{array}\right], \\
I_{1} & =\left[\begin{array}{ll}
I & -I
\end{array}\right], \\
I_{2} & =\left[\begin{array}{l}
0 \\
I
\end{array}\right], \\
\bar{C} & =\left[\begin{array}{ll}
0 & C
\end{array}\right], \\
\bar{F} & =\left[\begin{array}{c}
f\left(k, x_{k}\right) \\
F_{k}
\end{array}\right] .
\end{aligned}
$$

Definition 2 (see [13]). System (7) is stochastically stable if, for every finite $\eta_{0}$ and $\tau_{0} \in \Omega, d_{0} \in \Gamma$, there exists a finite matrix $R>0$ such that

$$
E\left\{\sum_{k=0}^{\infty}\left\|\eta_{k}\right\|^{2} \mid \eta_{0}, \tau_{0}, d_{0}\right\}<\eta_{0}^{T} R \eta_{0} .
$$

In this paper, our objective is to design the dynamic observerbased control scheme (3) and (4), such that the closedloop system (7) is stochastically stable on condition that the transition probabilities of $\tau_{k}$ and $d_{k}$ are both partly inaccessible.

\section{Main Results and Proofs}

In this section, we will present the main results. To proceed, we will need the following three lemmas.

Lemma 3 (see [19]). For any positive-definite matrix $W$, scalars $\delta$, $\delta_{0}$ satisfying $\delta \geq \delta_{0} \geq 1$ and vector function $v_{l}$, one has $\left(\sum_{l=\delta_{0}}^{\delta} v_{l}\right)^{T} W \sum_{l=\delta_{0}}^{\delta} v_{l} \leq\left(\delta-\delta_{0}+1\right) \sum_{l=\delta_{0}}^{\delta} v_{l}^{T} W v_{l}$.
Lemma 4 (see [20], S-procedure). Let $T_{l} \in R^{n \times n}(l=0,1$, $\ldots, z)$ be symmetric matrices. The conditions on $\xi^{T} T_{0} \xi<0$, $\forall \xi \neq 0$ s.t. $\xi^{T} T_{s} \xi \leq 0,(l=0,1, \ldots, z)$ hold if there exist scalars $\varepsilon_{l} \geq 0(l=0,1, \ldots, z)$ such that $T_{0}-\sum_{l=1}^{z} \varepsilon_{l} T_{l}<0$.

Lemma 5 (see [21]). For given scalars $\lambda_{i} \geq 0\left(i=1, \ldots, N_{1}\right)$, $\pi_{r} \geq 0\left(r=1, \ldots, N_{2}\right)$ and matrix $P_{i, r} \geq 0$, the following inequality always holds:

$$
\sum_{i=1}^{N_{1}} \sum_{r=1}^{N_{2}} \lambda_{i} \pi_{r} P_{i, r} \leq \sum_{i=1}^{N_{1}} \sum_{r=1}^{N_{2}} \lambda_{i} \pi_{r} \sum_{i=1}^{N_{1}} \sum_{r=1}^{N_{2}} P_{i, r}
$$

In the following, a sufficient condition such that the closed-loop system (7) is stochastically stable will be derived.

Theorem 6. Taking the controller gain matrix $K$ and observer gain matrix $L$, if there exist positive-definite matrices $P_{i, r}>0$, $P_{j, s}>0, Q_{1}>0, Q_{2}>0, Q_{3}>0, Q_{4}>0, Q_{5}>0, Q_{6}>0$, $Z_{1}>0, Z_{2}>0, Z_{3}>0, Z_{4}>0$ and scalar $\varepsilon_{1} \geq 0, \varepsilon_{2} \geq 0$ such that the following inequality

$$
\Phi \triangleq\left[\begin{array}{cccccccc}
\Phi_{11} & * & * & * & * & * & * & * \\
\Phi_{21} & \Phi_{22} & * & * & * & * & * & * \\
\Phi_{31} & \Phi_{32} & \Phi_{33} & * & * & * & * & * \\
\Phi_{41} & \Phi_{42} & \Phi_{43} & \Phi_{44} & * & * & * & * \\
Z_{2} & Z_{1} & 0 & 0 & \Phi_{55} & * & * & * \\
0 & Z_{1} & 0 & 0 & 0 & \Phi_{66} & * & * \\
Z_{4} & 0 & Z_{3} & 0 & 0 & 0 & \Phi_{77} & * \\
0 & 0 & Z_{3} & 0 & 0 & 0 & 0 & \Phi_{88}
\end{array}\right]<0,
$$

where

$$
\begin{aligned}
\Phi_{11} & =\left(d_{M}-d_{m}+1\right) Q_{1}+\left(\tau_{M}-\tau_{m}+1\right) Q_{2}+Q_{3} \\
+ & Q_{4}+Q_{5}+Q_{6}+\left(d_{M}-d_{m}\right)^{2}\left(\bar{A}-B_{1} K I_{1}-I\right)^{T} \\
\cdot & Z_{1}\left(\bar{A}-B_{1} K I_{1}-I\right)+d_{m}^{2}\left(\bar{A}-B_{1} K I_{1}-I\right)^{T} \\
\cdot & Z_{2}\left(\bar{A}-B_{1} K I_{1}-I\right)+\left(\tau_{M}-\tau_{m}\right)^{2} \\
\cdot & \left(\bar{A}-B_{1} K I_{1}-I\right)^{T} Z_{3}\left(\bar{A}-B_{1} K I_{1}-I\right) \\
+ & \tau_{m}^{2}\left(\bar{A}-B_{1} K I_{1}-I\right)^{T} Z_{4}\left(\bar{A}-B_{1} K I_{1}-I\right) \\
+ & \left(\bar{A}-B_{1} K I_{1}\right)^{T} \bar{P}_{j, s}\left(\bar{A}-B_{1} K I_{1}\right)-P_{i, r}+\varepsilon_{1} g^{2} I_{3}^{T} I_{3} \\
+ & \varepsilon_{2} g^{2} I_{4}^{T} I_{4}, \\
\Phi_{21} & =\left(d_{M}-d_{m}\right)^{2}\left(B_{2} K I_{1}\right)^{T} Z_{1}\left(\bar{A}-B_{1} K I_{1}-I\right) \\
& +d_{m}^{2}\left(B_{2} K I_{1}\right)^{T} Z_{2}\left(\bar{A}-B_{1} K I_{1}-I\right)+\left(\tau_{M}-\tau_{m}\right)^{2} \\
& \cdot\left(B_{2} K I_{1}\right)^{T} Z_{3}\left(\bar{A}-B_{1} K I_{1}-I\right)+\tau_{m}^{2}\left(B_{2} K I_{1}\right)^{T} \\
& \cdot Z_{4}\left(\bar{A}-B_{1} K I_{1}-I\right)+\left(B_{2} K I_{1}\right)^{T} \\
& \cdot \bar{P}_{j, s}\left(\bar{A}-B_{1} K I_{1}\right),
\end{aligned}
$$




$$
\begin{aligned}
& \Phi_{22}=\left(d_{M}-d_{m}\right)^{2}\left(B_{2} K I_{1}\right)^{T} Z_{1}\left(B_{2} K I_{1}\right) \\
& +d_{m}^{2}\left(B_{2} K I_{1}\right)^{T} Z_{2}\left(B_{2} K I_{1}\right)+\left(\tau_{M}-\tau_{m}\right)^{2}\left(B_{2} K I_{1}\right)^{T} \\
& \cdot Z_{3}\left(B_{2} K I_{1}\right)+\tau_{m}^{2}\left(B_{2} K I_{1}\right)^{T} Z_{4}\left(B_{2} K I_{1}\right) \\
& +\left(B_{2} K I_{1}\right)^{T} \bar{P}_{j, s}\left(B_{2} K I_{1}\right)-Q_{1}-2 Z_{1} \text {, } \\
& \Phi_{31}=-\left(d_{M}-d_{m}\right)^{2}\left(I_{2} L \bar{C}\right)^{T} Z_{1}\left(\bar{A}-B_{1} K I_{1}-I\right) \\
& -d_{m}^{2}\left(I_{2} L \bar{C}\right)^{T} Z_{2}\left(\bar{A}-B_{1} K I_{1}-I\right)-\left(\tau_{M}-\tau_{m}\right)^{2} \\
& \cdot\left(I_{2} L \bar{C}\right)^{T} Z_{3}\left(\bar{A}-B_{1} K I_{1}-I\right)-\tau_{m}^{2}\left(I_{2} L \bar{C}\right)^{T} \\
& \cdot Z_{4}\left(\bar{A}-B_{1} K I_{1}-I\right)-\left(I_{2} L \bar{C}\right)^{T} \bar{P}_{j, s}\left(\bar{A}-B_{1} K I_{1}\right) \text {, } \\
& \Phi_{32}=-\left(d_{M}-d_{m}\right)^{2}\left(I_{2} L \bar{C}\right)^{T} Z_{1}\left(B_{2} K I_{1}\right) \\
& -d_{m}^{2}\left(I_{2} L \bar{C}\right)^{T} Z_{2}\left(B_{2} K I_{1}\right)-\left(\tau_{M}-\tau_{m}\right)^{2}\left(I_{2} L \bar{C}\right)^{T} \\
& \cdot Z_{3}\left(B_{2} K I_{1}\right)-\tau_{m}^{2}\left(I_{2} L \bar{C}\right)^{T} Z_{4}\left(B_{2} K I_{1}\right)-\left(I_{2} L \bar{C}\right)^{T} \\
& \cdot \bar{P}_{j, s}\left(B_{2} K I\right) \text {, } \\
& \Phi_{33}=\left(d_{M}-d_{m}\right)^{2}\left(I_{2} L \bar{C}\right)^{T} Z_{1}\left(I_{2} L \bar{C}\right)+d_{m}^{2}\left(I_{2} L \bar{C}\right)^{T} \\
& \cdot Z_{2}\left(I_{2} L \bar{C}\right)+\left(\tau_{M}-\tau_{m}\right)^{2}\left(I_{2} L \bar{C}\right)^{T} Z_{3}\left(I_{2} L \bar{C}\right) \\
& +\tau_{m}^{2}\left(I_{2} L \bar{C}\right)^{T} Z_{4}\left(I_{2} L \bar{C}\right)+\left(I_{2} L \bar{C}\right)^{T} \bar{P}_{j, s}\left(I_{2} L \bar{C}\right) \\
& -Q_{2}-2 Z_{3} \\
& \Phi_{41}=\left(d_{M}-d_{m}\right)^{2} Z_{1}\left(\bar{A}-B_{1} K I_{1}-I\right) \\
& +d_{m}^{2} Z_{2}\left(\bar{A}-B_{1} K I_{1}-I\right)+\left(\tau_{M}-\tau_{m}\right)^{2} \\
& \cdot Z_{3}\left(\bar{A}-B_{1} K I_{1}-I\right)+\tau_{m}^{2} Z_{4}\left(\bar{A}-B_{1} K I_{1}-I\right) \\
& +\bar{P}_{j, s}\left(\bar{A}-B_{1} K I_{1}\right) \text {, } \\
& \Phi_{42}=\left(d_{M}-d_{m}\right)^{2} Z_{1}\left(B_{2} K I_{1}\right)+d_{m}^{2} Z_{2}\left(B_{2} K I_{1}\right) \\
& +\left(\tau_{M}-\tau_{m}\right)^{2} Z_{3}\left(B_{2} K I_{1}\right)+\tau_{m}^{2} Z_{4}\left(B_{2} K I_{1}\right) \\
& +\bar{P}_{j, s}\left(B_{2} K I_{1}\right) \text {, } \\
& \Phi_{43}=-\left(d_{M}-d_{m}\right)^{2} Z_{1}\left(I_{2} L \bar{C}\right)-d_{m}^{2} Z_{2}\left(I_{2} L \bar{C}\right) \\
& -\left(\tau_{M}-\tau_{m}\right)^{2} Z_{3}\left(I_{2} L \bar{C}\right)-\tau_{m}^{2} Z_{4}\left(I_{2} L \bar{C}\right) \\
& -\bar{P}_{j, s}\left(I_{2} L \bar{C}\right) \text {, } \\
& \Phi_{44}=\left(d_{M}-d_{m}\right)^{2} Z_{1}+d_{m}^{2} Z_{2}+\left(\tau_{M}-\tau_{m}\right)^{2} Z_{3} \\
& +\tau_{m}^{2} Z_{4}+\bar{P}_{j, s}-\varepsilon_{1} I_{3}^{T} I_{3}-\varepsilon_{2} I_{4}^{T} I_{4} \\
& \Phi_{55}=-Q_{3}-Z_{2}-Z_{1} \text {, }
\end{aligned}
$$$$
\begin{aligned}
& \Phi_{66}=-Q_{4}-Z_{1}, \\
& \Phi_{77}=-Q_{5}-Z_{4}-Z_{3}, \\
& \Phi_{88}=-Q_{6}-Z_{3}, \\
& I_{3}=\left[\begin{array}{ll}
I & 0
\end{array}\right], \\
& I_{4}=\left[\begin{array}{ll}
0 & I
\end{array}\right], \\
& \bar{P}_{j, s}=\sum_{j=\tau_{m}}^{\tau_{M}} \sum_{s=d_{m}}^{d_{M}} \lambda_{i j} \pi_{r s} P_{j, s},
\end{aligned}
$$

holds for all $i, j \in \Omega$ and $r, s \in \Gamma$, the closed-loop system (7) is stochastically stable.

Proof. Choose the Lyapunov function candidate as $V\left(\eta_{k}, \tau_{k}, d_{k}\right)=\sum_{\rho=1}^{5} V_{\rho}\left(\eta_{k}, \tau_{k}, d_{k}\right)$, where

$$
\begin{aligned}
& V_{1}\left(\eta_{k}, \tau_{k}, d_{k}\right)=\eta_{k}^{T} P_{\tau_{k}, d_{k}} \eta_{k}, \\
& V_{2}\left(\eta_{k}, d_{k}, \tau_{k}\right)=\sum_{l=k-d_{k}}^{k-1} \eta_{l}^{T} Q_{1} \eta_{l}+\sum_{l=k-\tau_{k}}^{k-1} \eta_{l}^{T} Q_{2} \eta_{l}, \\
& V_{3}\left(\eta_{k}, d_{k}, \tau_{k}\right)=\sum_{l=k-d_{m}}^{k-1} \eta_{l}^{T} Q_{3} \eta_{l}+\sum_{l=k-d_{M}}^{k-1} \eta_{l}^{T} Q_{4} \eta_{l} \\
& +\sum_{l=k-\tau_{m}}^{k-1} \eta_{l}^{T} Q_{5} \eta_{l}+\sum_{l=k-\tau_{M}}^{k-1} \eta_{l}^{T} Q_{6} \eta_{l} \\
& V_{4}\left(\eta_{k}, d_{k}, \tau_{k}\right)=\sum_{n=-d_{M}+1}^{-d_{m}} \sum_{l=k+n}^{k-1} \eta_{l}^{T} Q_{1} \eta_{l} \\
& +\sum_{n=-\tau_{M}+1}^{-\tau_{m}} \sum_{l=k+n}^{k-1} \eta_{l}^{T} Q_{2} \eta_{l} \\
& V_{5}\left(\eta_{k}, d_{k}, \tau_{k}\right)=\sum_{n=-d_{M}+1}^{-d_{m}} \sum_{l=k+n}^{k-1}\left(d_{M}-d_{m}\right) \zeta_{l}^{T} Z_{1} \zeta_{l} \\
& +\sum_{n=-d_{m}}^{-1} \sum_{l=k+n}^{k-1} d_{m} \zeta_{l}^{T} Z_{2} \zeta_{l} \\
& +\sum_{n=-\tau_{M}+1}^{-\tau_{m}} \sum_{l=k+n}^{k-1}\left(\tau_{M}-\tau_{m}\right) \zeta_{l}^{T} Z_{3} \zeta_{l} \\
& +\sum_{n=-\tau_{m}}^{-1} \sum_{l=k+n}^{k-1} \tau_{m} \zeta_{l}^{T} Z_{4} \zeta_{l} \\
& \zeta_{l}=\eta_{l+1}-\eta_{l} .
\end{aligned}
$$

In the following, we denote $P_{d_{k}, \tau_{k}}$ as $P_{r, i}$ when $d_{k}=r$ and $\tau_{k}=i$. Then along the solution of system (7), we have

$$
\begin{gathered}
E\left\{\Delta V_{1}\right\}=E\left\{\eta_{k+1}^{T} P_{\tau_{k+1}, d_{k+1}} \eta_{k+1} \mid \tau_{k}=i, d_{k}=r\right\} \\
-\eta_{k}^{T} P_{i, r} \eta_{k}=\left(\left(\bar{A}-B_{1} K I_{1}\right) \eta_{k}+B_{2} K I_{1} \eta_{k-d_{k}}\right.
\end{gathered}
$$




$$
\begin{aligned}
& \left.-I_{2} L \bar{C} \eta_{k-\tau_{k}}+\bar{F}_{k}\right)^{T} \sum_{j=\tau_{m}}^{\tau_{M}} \sum_{s=d_{m}}^{d_{M}} \lambda_{i j} \pi_{r s} P_{j, s} \\
& \cdot\left(\left(\bar{A}-B_{1} K I_{1}\right) \eta_{k}+B_{2} K I_{1} \eta_{k-d_{k}}-I_{2} L \bar{C} \eta_{k-\tau_{k}}+\bar{F}_{k}\right) \\
& -\eta_{k}^{T} P_{i, r} \eta_{k}, \\
& E\left\{\Delta V_{2}\right\}=\eta_{k}^{T} Q_{1} \eta_{k}-\eta_{k-r}^{T} Q_{1} \eta_{k-r}+\sum_{l=k+1-d_{k+1}}^{k-1} \eta_{l}^{T} Q_{1} \eta_{l} \\
& -\sum_{l=k+1-d_{k}}^{k-1} \eta_{l}^{T} Q_{1} \eta_{l}+\eta_{k}^{T} Q_{2} \eta_{k}-\eta_{k-i}^{T} Q_{2} \eta_{k-i} \\
& +\sum_{l=k+1-\tau_{k+1}}^{k-1} \eta_{l}^{T} Q_{2} \eta_{l}-\sum_{l=k+1-\tau_{k}}^{k-1} \eta_{l}^{T} Q_{2} \eta_{l} .
\end{aligned}
$$

It is noticed that

$$
\begin{aligned}
\sum_{l=k+1-d_{k+1}}^{k-1} \eta_{l}^{T} Q_{1} \eta_{l} & =\sum_{l=k+1-d_{m}}^{k-1} \eta_{l}^{T} Q_{1} \eta_{l}+\sum_{l=k+1-d_{k+1}}^{k-d_{m}} \eta_{l}^{T} Q_{1} \eta_{l} \\
& \leq \sum_{l=k+1-d_{k}}^{k-1} \eta_{l}^{T} Q_{1} \eta_{l}+\sum_{l=k+1-d_{M}}^{k-d_{m}} \eta_{l}^{T} Q_{1} \eta_{l}, \\
\sum_{l=k+1-\tau_{k+1}}^{k-1} \eta_{l}^{T} Q_{2} \eta_{l} & =\sum_{l=k+1-\tau_{m}}^{k-1} \eta_{l}^{T} Q_{2} \eta_{l}+\sum_{l=k+1-\tau_{k+1}}^{k-\tau_{m}} \eta_{l}^{T} Q_{2} \eta_{l} \\
& \leq \sum_{l=k+1-\tau_{k}}^{k-1} \eta_{l}^{T} Q_{2} \eta_{l}+\sum_{l=k+1-\tau_{M}}^{k-\tau_{m}} \eta_{l}^{T} Q_{2} \eta_{l} .
\end{aligned}
$$

Therefore, we obtain

$$
\begin{aligned}
E\left\{\Delta V_{2}\right\} \leq & \eta_{k}^{T} Q_{1} \eta_{k}-\eta_{k-r}^{T} Q_{1} \eta_{k-r}+\sum_{l=k+1-d_{M}}^{k-d_{m}} \eta_{l}^{T} Q_{1} \eta_{l} \\
& +\eta_{k}^{T} Q_{2} \eta_{k}-\eta_{k-i}^{T} Q_{2} \eta_{k-i} \\
& +\sum_{l=k+1-\tau_{M}}^{k-\tau_{m}} \eta_{l}^{T} Q_{2} \eta_{l}, \\
E\left\{\Delta V_{3}\right\}= & \eta_{k}^{T} Q_{3} \eta_{k}-\eta_{k-d_{m}}^{T} Q_{3} \eta_{k-d_{m}}+\eta_{k}^{T} Q_{4} \eta_{k} \\
& -\eta_{k-d_{M}}^{T} Q_{4} \eta_{k-d_{M}}+\eta_{k}^{T} Q_{5} \eta_{k} \\
& -\eta_{k-\tau_{m}}^{T} Q_{5} \eta_{k-\tau_{m}}+\eta_{k}^{T} Q_{6} \eta_{k} \\
& -\eta_{k-\tau_{M}}^{T} Q_{6} \eta_{k-\tau_{M}}, \\
E\left\{\Delta V_{4}\right\}= & \left(d_{M}-d_{m}\right) \eta_{k}^{T} Q_{1} \eta_{k}-\sum_{l=k+1-d_{M}}^{k-d_{m}} \eta_{l}^{T} Q_{1} \eta_{l} \\
& +\left(\tau_{M}-\tau_{m}\right) \eta_{k}^{T} Q_{2} \eta_{k}-\sum_{l=k+1-\tau_{M}}^{k-\tau_{m}} \eta_{l}^{T} Q_{2} \eta_{l},
\end{aligned}
$$

$$
\begin{aligned}
E\left\{\Delta V_{5}\right\}= & \left(d_{M}-d_{m}\right)^{2} \zeta_{k}^{T} Z_{1} \zeta_{k} \\
& -\sum_{l=k-d_{M}}^{k-d_{m}-1}\left(d_{M}-d_{m}\right) \zeta_{l}^{T} Z_{1} \zeta_{l} \\
& +d_{m}^{2} \zeta_{k}^{T} Z_{2} \zeta_{k}-d_{m} \sum_{l=k-d_{m}}^{k-1} \zeta_{l}^{T} Z_{2} \zeta_{l} \\
& +\left(\tau_{M}-\tau_{m}\right)^{2} \zeta_{k}^{T} Z_{3} \zeta_{k} \\
& -\sum_{l=k-\tau_{M}}^{k-\tau_{m}-1}\left(\tau_{M}-\tau_{m}\right) \zeta_{l}^{T} Z_{3} \zeta_{l}+\tau_{m}^{2} \zeta_{k}^{T} Z_{4} \zeta_{k} \\
& -\tau_{m} \sum_{l=k-\tau_{m}}^{k-1} \zeta_{l}^{T} Z_{4} \zeta_{l} .
\end{aligned}
$$

Note that

$$
\begin{aligned}
- & d_{m} \\
l=k-d_{m} & \sum_{l}^{k-1} Z_{2} \zeta_{l}-\sum_{l=k-d_{M}}^{k-d_{m}-1}\left(d_{M}-d_{m}\right) \zeta_{l}^{T} Z_{1} \zeta_{l} \\
= & -d_{m} \sum_{l=k-d_{m}}^{k-1} \zeta_{l}^{T} Z_{2} \zeta_{l}-\sum_{l=k-r}^{k-d_{m}-1}\left(d_{M}-d_{m}\right) \zeta_{l}^{T} Z_{1} \zeta_{l} \\
& -\sum_{l=k-d_{M}}^{k-r-1}\left(d_{M}-d_{m}\right) \zeta_{l}^{T} Z_{1} \zeta_{l} \\
\leq & -d_{m} \sum_{l=k-d_{m}}^{k-1} \zeta_{l}^{T} Z_{2} \zeta_{l}-\sum_{l=k-r}^{k-d_{m}-1}\left(r-d_{m}\right) \zeta_{l}^{T} Z_{1} \zeta_{l} \\
& -\sum_{l=k-d_{M}}^{k-r-1}\left(d_{M}-r\right) \zeta_{l}^{T} Z_{1} \zeta_{l} .
\end{aligned}
$$

We can obtain the following inequality by Lemma 3:

$$
\begin{aligned}
-d_{m} & \sum_{l=k-d_{m}}^{k-1} \zeta_{l}^{T} Z_{2} \zeta_{l}-\sum_{l=k-d_{M}}^{k-d_{m}-1}\left(d_{M}-d_{m}\right) \zeta_{l}^{T} Z_{1} \zeta_{l} \\
\leq & -\left[\eta_{k}-\eta_{k-d_{m}}\right]^{T} Z_{2}\left[\eta_{k}-\eta_{k-d_{m}}\right] \\
- & {\left[\eta_{k-d_{m}}-\eta_{k-r}\right]^{T} Z_{1}\left[\eta_{k-d_{m}}-\eta_{k-r}\right] } \\
- & {\left[\eta_{k-r}-\eta_{k-d_{M}}\right]^{T} Z_{1}\left[\eta_{k-r}-\eta_{k-d_{M}}\right] . }
\end{aligned}
$$

Similarly, we have

$$
\begin{aligned}
-\tau_{m} & \sum_{l=k-\tau_{m}}^{k-1} \zeta_{l}^{T} Z_{4} \zeta_{l}-\sum_{l=k-\tau_{M}}^{k-\tau_{m}-1}\left(\tau_{M}-\tau_{m}\right) \zeta_{l}^{T} Z_{3} \zeta_{l} \\
\leq & -\left[\eta_{k}-\eta_{k-\tau_{m}}\right]^{T} Z_{4}\left[\eta_{k}-\eta_{k-\tau_{m}}\right] \\
& -\left[\eta_{k-\tau_{m}}-\eta_{k-i}\right]^{T} Z_{3}\left[\eta_{k-\tau_{m}}-\eta_{k-i}\right] \\
& -\left[\eta_{k-i}-\eta_{k-\tau_{M}}\right]^{T} Z_{3}\left[\eta_{k-i}-\eta_{k-\tau_{M}}\right]
\end{aligned}
$$


From (14)-(19), we have

$$
E\left\{\Delta V\left(\eta_{k}, d_{k}, \tau_{k}\right) \mid \tau_{k}=i, d_{k}=r\right\} \leq \xi_{k}^{T} \bar{\Phi} \xi_{k},
$$

where

$$
\begin{aligned}
\bar{\Phi} & =\left[\begin{array}{cccccccc}
\bar{\Phi}_{11} & * & * & * & * & * & * & * \\
\Phi_{21} & \Phi_{22} & * & * & * & * & * & * \\
\Phi_{31} & \Phi_{32} & \Phi_{33} & * & * & * & * & * \\
\Phi_{41} & \Phi_{42} & \Phi_{43} & \bar{\Phi}_{44} & * & * & * & * \\
Z_{2} & Z_{1} & 0 & 0 & \Phi_{55} & * & * & * \\
0 & Z_{1} & 0 & 0 & 0 & \Phi_{66} & * & * \\
Z_{4} & 0 & Z_{3} & 0 & 0 & 0 & \Phi_{77} & * \\
0 & 0 & Z_{3} & 0 & 0 & 0 & 0 & \Phi_{88}
\end{array}\right], \\
\bar{\Phi}_{11} & =\left(d_{M}-d_{m}+1\right) Q_{1}+\left(\tau_{M}-\tau_{m}+1\right) Q_{2}+Q_{3} \\
& +Q_{4}+Q_{5}+Q_{6}+\left(d_{M}-d_{m}\right)^{2}\left(\bar{A}-B_{1} K I_{1}-I\right)^{T} \\
& \cdot Z_{1}\left(\bar{A}-B_{1} K I_{1}-I\right)+d_{m}^{2}\left(\bar{A}-B_{1} K I_{1}-I\right)^{T} \\
& \cdot Z_{2}\left(\bar{A}-B_{1} K I_{1}-I\right)+\left(\tau_{M}-\tau_{m}\right)^{2} \\
& +\left(\bar{A}-B_{1} K I_{1}-I\right)^{T} Z_{3}\left(\bar{A}-B_{1} K I_{1}-I\right) \\
& +\left(\bar{A}-B_{1} K I_{1}\right)^{T} \bar{P}_{j, s}\left(\bar{A}-B_{1} K I_{1}\right)-P_{i, r}, \\
\bar{\Phi}_{44} & =\left(d_{M}-d_{m}\right)^{2} Z_{1}+d_{m}^{2} Z_{2}+\left(\tau_{M}-\tau_{m}\right)^{2} Z_{3} \\
& {\left[\eta_{k}^{T} \eta_{k-r}^{T} \eta_{k-i}^{T} B_{1} K I_{1}-I\right)^{T} Z_{4}\left(\bar{A}-B_{1} K I_{1}-I\right) }
\end{aligned}
$$

It follows from (2) that

$$
\begin{aligned}
f_{k, x_{k}}^{T} f_{k, x_{k}} & \leq g^{2} x_{k}^{T} x_{k}, \\
F_{k}^{T} F_{k} & \leq g^{2} e_{k}^{T} e_{k},
\end{aligned}
$$

which imply that

$$
\begin{aligned}
& f_{k, x_{k}}^{T} f_{k, x_{k}}-g^{2} x_{k}^{T} x_{k}=\bar{F}_{k}^{T} I_{3}^{T} I_{3} \bar{F}_{k}-g^{2} \eta_{k}^{T} I_{3}^{T} I_{3} \eta_{k} \\
& =\xi_{k}^{T}\left[\begin{array}{cccccccc}
-g^{2} I_{3}^{T} I_{3} & * & * & * & * & * & * & * \\
0 & 0 & * & * & * & * & * & * \\
0 & 0 & 0 & * & * & * & * & * \\
0 & 0 & 0 & I_{3}^{T} I_{3} & * & * & * & * \\
0 & 0 & 0 & 0 & 0 & * & * & * \\
0 & 0 & 0 & 0 & 0 & 0 & * & * \\
0 & 0 & 0 & 0 & 0 & 0 & 0 & * \\
0 & 0 & 0 & 0 & 0 & 0 & 0 & 0
\end{array}\right] \xi_{k}
\end{aligned}
$$

$$
\triangleq \xi_{k}^{T} \Lambda_{1} \xi_{k} \leq 0
$$$$
F_{k}^{T} F_{k}-g^{2} e_{k}^{T} e_{k}=\bar{F}_{k}^{T} I_{4}^{T} I_{4} \bar{F}_{k}-g^{2} \eta_{k}^{T} I_{4}^{T} I_{4} \eta_{k}
$$$$
=\xi_{k}^{T}\left[\begin{array}{cccccccc}
-g^{2} I_{4}^{T} I_{4} & * & * & * & * & * & * & * \\
0 & 0 & * & * & * & * & * & * \\
0 & 0 & 0 & * & * & * & * & * \\
0 & 0 & 0 & I_{4}^{T} I_{4} & * & * & * & * \\
0 & 0 & 0 & 0 & 0 & * & * & * \\
0 & 0 & 0 & 0 & 0 & 0 & * & * \\
0 & 0 & 0 & 0 & 0 & 0 & 0 & * \\
0 & 0 & 0 & 0 & 0 & 0 & 0 & 0
\end{array}\right] \xi_{k}
$$

$$
\triangleq \xi_{k}^{T} \Lambda_{2} \xi_{k} \leq 0 \text {. }
$$

By the well-known S-procedure, that is, Lemma 4, we can get $E\left\{\Delta V\left(\eta_{k}, d_{k}, \tau_{k}\right)\right\} \leq \xi_{k}^{T} \bar{\Phi} \xi_{k}<0$ with constraints (23) holding if there exist nonnegative real scalars $\varepsilon_{1} \geq 0, \varepsilon_{2} \geq 0$ such that

$$
\bar{\Phi}-\varepsilon_{1} \Lambda_{1}-\varepsilon_{2} \Lambda_{2}=\Phi<0 .
$$

From (24), we have

$$
\begin{aligned}
E\{ & \left.\Delta V\left(\eta_{k}, d_{k}, \tau_{k}\right) \mid \tau_{k}=i, d_{k}=r\right\} \leq-\lambda_{\min }(-\Phi) \\
& \cdot \xi_{k}^{T} \xi_{k}=-\lambda_{\min }(-\Phi)\left(\eta_{k}^{T} \eta_{k}+\eta_{k-r}^{T} \eta_{k-r}+\eta_{k-i}^{T} \eta_{k-i}\right. \\
& +\bar{F}_{k}^{T} \bar{F}_{k}+\eta_{k-d_{m}}^{T} \eta_{k-d_{m}}+\eta_{k-d_{M}}^{T} \eta_{k-d_{M}}+\eta_{k-\tau_{m}}^{T} \eta_{k-\tau_{m}} \\
& \left.+\eta_{k-\tau_{M}}^{T} \eta_{k-\tau_{M}}\right) \leq-\alpha\left\|\eta_{k}\right\|^{2},
\end{aligned}
$$

where $\alpha=\inf \left\{-\lambda_{\min }(-\Phi)\right\}>0$.

From (25), we can see that, for any $T \geq 1$,

$$
\begin{aligned}
E\left\{\sum_{k=0}^{\infty}\left\|\eta_{k}\right\|^{2}\right\} \leq & \frac{1}{\alpha} E\left\{V\left(\eta_{0}, d_{0}, \tau_{0}\right)\right\} \\
& -\frac{1}{\alpha} E\left\{V\left(\eta_{T+1}, d_{T+1}, \tau_{T+1}\right)\right\} \\
\leq & \frac{1}{\alpha} E\left\{V\left(\eta_{0}, d_{0}, \tau_{0}\right)\right\} .
\end{aligned}
$$

According to Definition 2, the closed-loop system (7) is stochastically stable, which completes the proof.

Theorem 7. Taking the controller gain matrix $K$ and observer gain matrix $L$, the closed-loop system (7) is stochastically stable if there exist positive-definite matrices $P_{i, r}>0, P_{j, s}>0, F_{j, s}>$ $0, Q_{1}>0, Q_{2}>0, Q_{3}>0, Q_{4}>0, Q_{5}>0, Q_{6}>0, Z_{1}>0$, $Z_{2}>0, Z_{3}>0, Z_{4}>0, Y_{1}>0, Y_{2}>0, Y_{3}>0, Y_{4}>0$ and scalar $\varepsilon_{1} \geq 0, \varepsilon_{2} \geq 0$ such that

$$
\begin{aligned}
& {\left[\begin{array}{cccc}
\Lambda_{11} & * & * & * \\
\Lambda_{21} & \Lambda_{22} & * & * \\
\Lambda_{31} & 0 & \Lambda_{33} & * \\
\Lambda_{41} & 0 & 0 & \Lambda_{44}
\end{array}\right]<0, } \\
& \\
& P_{j, s} F_{j, s}=I, \\
& Y_{l} Z_{l}=I, \quad l \in\{1,2,3,4\},
\end{aligned}
$$


where

$$
\begin{aligned}
& \Lambda_{11}=\left[\begin{array}{cccc}
\bar{\Lambda}_{11} & * & * & * \\
0 & -Q_{1}-2 Z_{1} & * & * \\
0 & 0 & -Q_{2}-2 Z_{3} & * \\
0 & 0 & 0 & -\tau_{1} I_{3}^{T} I_{3}-\tau_{2} I_{4}^{T} I_{4}
\end{array}\right] \text {, } \\
& \Lambda_{21}=\left[\begin{array}{cccc}
Z_{2} & Z_{1} & 0 & 0 \\
0 & Z_{1} & 0 & 0 \\
Z_{4} & 0 & Z_{3} & 0 \\
0 & 0 & Z_{3} & 0
\end{array}\right] \\
& \Lambda_{22}=\left[\begin{array}{cccc}
-Q_{3}-Z_{2}-Z_{1} & * & * & * \\
0 & -Q_{4}-Z_{1} & * & * \\
0 & 0 & -Q_{5}-Z_{4}-Z_{3} & * \\
0 & 0 & 0 & -Q_{6}-Z_{3}
\end{array}\right] \text {, } \\
& \Lambda_{31}=\left[\begin{array}{cccc}
\left(d_{M}-d_{m}\right) \Upsilon & \left(d_{M}-d_{m}\right) B_{2} K I_{1} & -\left(d_{M}-d_{m}\right) I_{2} L \bar{C} & \left(d_{M}-d_{m}\right) I \\
d_{m} \Upsilon & d_{m} B_{2} K I_{1} & -d_{m} I_{2} L \bar{C} & -d_{m} I \\
\left(\tau_{M}-\tau_{m}\right) \Upsilon & \left(\tau_{M}-\tau_{m}\right) B_{2} K I_{1} & -\left(\tau_{M}-\tau_{m}\right) I_{2} L \bar{C} & \left(\tau_{M}-\tau_{m}\right) I \\
\tau_{m} \Upsilon & \tau_{m} B_{2} K I_{1} & -\tau_{m} I_{2} L \bar{C} & -\tau_{m} I
\end{array}\right], \\
& \Lambda_{33}=\left[\begin{array}{cccc}
-Y_{1} & * & * & * \\
0 & -Y_{2} & * & * \\
0 & 0 & -Y_{3} & * \\
0 & 0 & 0 & -Y_{4}
\end{array}\right]
\end{aligned}
$$

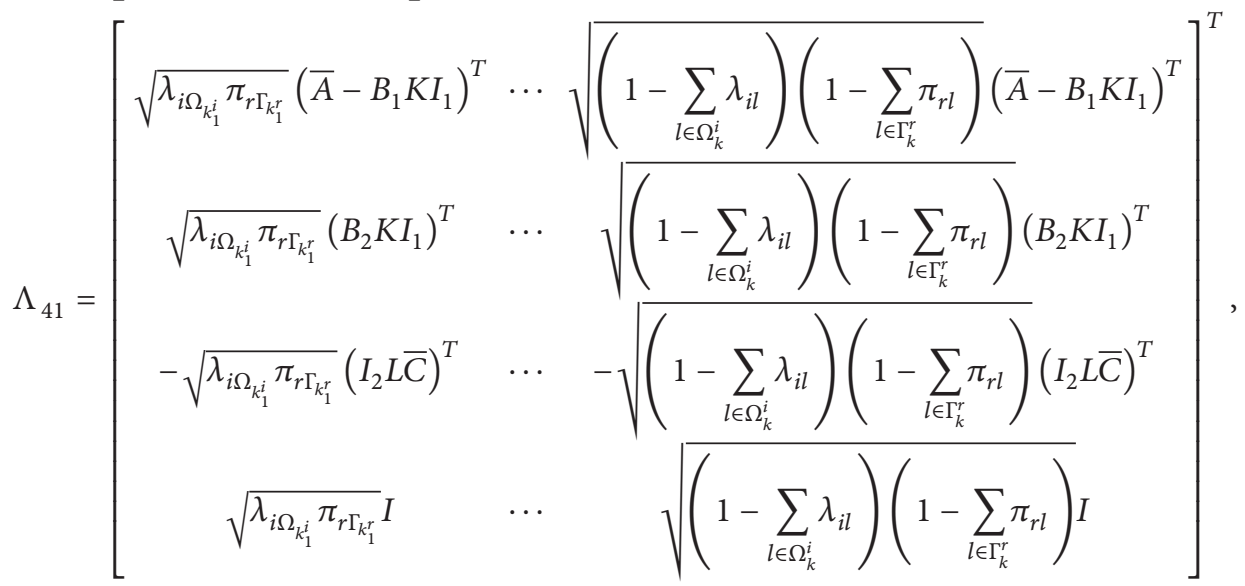

$$
\begin{aligned}
& \Lambda_{44}=\left[\begin{array}{ccc}
-F_{\Omega_{k_{1}^{i}}, \Gamma_{k_{1}^{r}}} & * & * \\
0 & \ddots & * \\
0 & 0 & -F_{\Omega_{\bar{k}_{e-\mu}^{i}},}, \Gamma_{\bar{k}_{p-\theta}^{r}}^{r}
\end{array}\right] \text {, } \\
& \bar{\Lambda}_{11}=\left(d_{M}-d_{m}+1\right) Q_{1}+\left(\tau_{M}-\tau_{m}+1\right) Q_{2}+Q_{3}+Q_{4}+Q_{5}+Q_{6}-Z_{2}-Z_{4}+\varepsilon_{1} g^{2} I_{3}^{T} I_{3}+\varepsilon_{2} g^{2} I_{4}^{T} I_{4}-P_{i, r}, \\
& \Upsilon=\bar{A}-B_{1} K I_{1}-I \\
& \sum_{j \in \Omega_{k}^{i}} \sum_{s \in \Gamma_{u k}^{r}} \lambda_{i j} \pi_{r s} P_{j, s} \leq\left(1-\sum_{l \in \Gamma_{k}^{r}} \pi_{r l}\right) \sum_{j \in \Omega_{k}^{i}} \lambda_{i j} \sum_{j \in \Omega_{k}^{i}} \sum_{s \in \Gamma_{u k}^{r}} P_{j, s}, \\
& \sum_{j \in \Omega_{u k}^{i}} \sum_{s \in \Gamma_{u k}^{r}} \lambda_{i j} \pi_{r s} P_{j, s} \\
& \leq\left(1-\sum_{l \in \Omega_{k}^{i}} \lambda_{i l}\right) \sum_{s \in \Gamma_{k}^{r}} \pi_{r s} \sum_{j \in \Omega_{u k}^{i}} \sum_{s \in \Gamma_{k}^{r}} P_{j, s}, \\
& \leq\left(1-\sum_{l \in \Gamma_{k}^{r}} \pi_{r l}\right)\left(1-\sum_{l \in \Omega_{k}^{i}} \lambda_{i l}\right) \sum_{j \in \Omega_{u k}^{i}} \sum_{s \in \Gamma_{u k}^{r}} P_{j, s} .
\end{aligned}
$$

hold for all $i, j \in \Omega$ and $r, s \in \Gamma$.

Proof. Making use of Lemma 5, we have 
Letting $P_{j, s}^{-1}=F_{j, s}, j \in \Omega, s \in \Gamma, Z_{l}^{-1}=Y_{l}, l \in\{1,2,3,4\}$ and by Schur complement Lemma, (27) can be obtained from (11) and (30)-(32), which complete the proof.

In Theorem 7, in order to get a desired controller (3)(4) for the closed-loop NCS (7) with partly inaccessible transition probabilities, Lemma 5 is used to separate $\lambda_{i j} \pi_{r s}$ from $\lambda_{i j} \pi_{r s} P_{j, s}$. However, this could lead to conservativeness to some extents as shown in the Numerical Example. In the following, a less conservative result will be given in the following theorem.

Theorem 8. There exists dynamic observer-based control scheme (3)-(4) such that the resulting closed-loop system (7) is stochastically stable if, for all $i, j \in \Omega$ and $r, s \in \Gamma$, there exist matrices $P_{i, r}>0, P_{j, s}>0, F_{j, s}>0, Q_{1}>0, Q_{2}>0, Q_{3}>0$, $Q_{4}>0, Q_{5}>0, Q_{6}>0, Z_{1}>0, Z_{2}>0, Z_{3}>0, Z_{4}>0, Y_{1}>$ $0, Y_{2}>0, Y_{3}>0, Y_{4}>0$ and scalar $\varepsilon_{1} \geq 0, \varepsilon_{2} \geq 0$ such that

$$
\left[\begin{array}{cc}
\sum_{j \in \Omega_{k}^{i}} \sum_{s \in \Gamma_{k}^{r}} \lambda_{i j} \pi_{r s} \Lambda & * \\
\Theta_{\Omega_{k}^{i}, r_{k}^{r}} & -\Delta_{\Omega_{k}^{i}, \Gamma_{k}^{r}}
\end{array}\right]<0,
$$

$$
\begin{aligned}
& {\left[\begin{array}{cc}
\sum_{s \in \Gamma_{k}^{r}} \lambda_{i j} \pi_{r s} \Lambda & * \\
\Theta_{\Omega_{u k}^{i}, \Gamma_{k}^{r}} & -\Delta_{\Omega_{u k}^{i}, \Gamma_{k}^{r}}
\end{array}\right]<0, \quad \forall j \in \Omega_{u k}^{i},} \\
& {\left[\begin{array}{cc}
\sum_{j \in \Omega_{k}^{i}} \lambda_{i j} \Lambda & * \\
\Theta_{\Omega_{k}^{i}, \Gamma_{u k}^{r}} & -\Delta_{\Omega_{k}^{i}, \Gamma_{u k}^{r}}^{r}
\end{array}\right]<0, \quad \forall s \in \Gamma_{u k}^{r},} \\
& {\left[\begin{array}{cc}
\Lambda & * \\
\Theta_{\Omega_{u k}^{i}, \Gamma_{u k}^{r}} & -\Delta_{\Omega_{u k}^{i}, \Gamma_{u k}^{r}}^{r}
\end{array}\right]<0,} \\
& F_{j, s} P_{j, s}=I, \quad \forall j \in \Omega_{u k}^{i}, \quad \forall s \in \Gamma_{u k}^{r}, \\
& Y_{l} Z_{l}=I, \quad l \in\{1,2,3,4\},
\end{aligned}
$$

where

$$
\begin{aligned}
& \Lambda=\left[\begin{array}{ccc}
\Lambda_{11} & * & * \\
\Lambda_{21} & \Lambda_{22} & * \\
\Lambda_{31} & 0 & \Lambda_{33}
\end{array}\right], \\
& \Lambda_{11}=\left[\begin{array}{cccc}
\bar{\Lambda}_{11} & * & * & * \\
0 & -Q_{1}-2 Z_{1} & * & * \\
0 & 0 & -Q_{2}-2 Z_{3} & * \\
0 & 0 & 0 & -\tau_{1} I_{3}^{T} I_{3}-\tau_{2} I_{4}^{T} I_{4}
\end{array}\right] \text {, } \\
& \Lambda_{21}=\left[\begin{array}{cccc}
Z_{2} & Z_{1} & 0 & 0 \\
0 & Z_{1} & 0 & 0 \\
Z_{4} & 0 & Z_{3} & 0 \\
0 & 0 & Z_{3} & 0
\end{array}\right] \\
& \Lambda_{22}=\left[\begin{array}{cccc}
-Q_{3}-Z_{2}-Z_{1} & * & * & * \\
0 & -Q_{4}-Z_{1} & * & * \\
0 & 0 & -Q_{5}-Z_{4}-Z_{3} & * \\
0 & 0 & 0 & -Q_{6}-Z_{3}
\end{array}\right] \text {, } \\
& \Lambda_{31}=\left[\begin{array}{cccc}
\left(d_{M}-d_{m}\right) \Upsilon & \left(d_{M}-d_{m}\right) B_{2} K I_{1} & -\left(d_{M}-d_{m}\right) I_{2} L \bar{C} & \left(d_{M}-d_{m}\right) I \\
d_{m} \Upsilon & d_{m} B_{2} K I_{1} & -d_{m} I_{2} L \bar{C} & -d_{m} I \\
\left(\tau_{M}-\tau_{m}\right) \Upsilon & \left(\tau_{M}-\tau_{m}\right) B_{2} K I_{1} & -\left(\tau_{M}-\tau_{m}\right) I_{2} L \bar{C} & \left(\tau_{M}-\tau_{m}\right) I \\
\tau_{m} \Upsilon & \tau_{m} B_{2} K I_{1} & -\tau_{m} I_{2} L \bar{C} & -\tau_{m} I
\end{array}\right] \text {, } \\
& \Lambda_{33}=\left[\begin{array}{cccc}
-Y_{1} & * & * & * \\
0 & -Y_{2} & * & * \\
0 & 0 & -Y_{3} & * \\
0 & 0 & 0 & -Y_{4}
\end{array}\right]
\end{aligned}
$$




$$
\begin{aligned}
& \bar{\Lambda}_{11}=\left(d_{M}-d_{m}+1\right) Q_{1}+\left(\tau_{M}-\tau_{m}+1\right) Q_{2}+Q_{3}+Q_{4}+Q_{5}+Q_{6}-Z_{2}-Z_{4}+\varepsilon_{1} g^{2} I_{3}^{T} I_{3}+\varepsilon_{2} g^{2} I_{4}^{T} I_{4}-P_{i, r}
\end{aligned}
$$

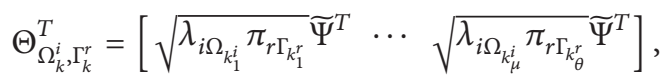

$$
\begin{aligned}
& \Delta_{\Omega_{k^{i}}^{i}, \Gamma_{k}^{r}}=\operatorname{diag}\left\{-F_{\Omega_{k_{1}^{i}} \Gamma_{k_{1}^{r}}^{r}}, \ldots,-F_{\Omega_{k_{k}^{i}} \Gamma_{\theta}^{r}}\right\} \text {, }
\end{aligned}
$$

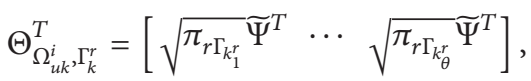

$$
\begin{aligned}
& \Delta_{\Omega_{u k}^{i}, \Gamma_{k}^{r}}=\operatorname{diag}\left\{-F_{j, \Gamma_{1}^{r}}, \ldots,-F_{j, \Gamma_{k_{\theta}^{r}}}\right\} \text {, } \\
& \Theta_{\Omega_{k}^{i}, \Gamma_{u k}^{r}}^{T}=\left[\begin{array}{lll}
\sqrt{\lambda_{i \Omega_{k_{1}^{i}}}} \widetilde{\Psi}^{T} \cdots \sqrt{\lambda_{i \Omega_{k_{\mu}^{i}}}} \widetilde{\Psi}^{T}
\end{array}\right] \\
& \Delta_{\Omega_{k}^{i}, \Gamma_{u k}^{r}}=\operatorname{diag}\left\{-F_{\Omega_{k_{1}^{i}}, s}, \ldots,-F_{\Omega_{k_{\mu}^{i}}^{i}, s}\right\} \text {, } \\
& \Theta_{\Omega_{u k}^{i}, \Gamma_{u k}^{r}}^{T}=\left[\begin{array}{lll}
\widetilde{\Psi}^{T} & \ldots & \widetilde{\Psi}^{T}
\end{array}\right], \\
& \Delta_{\Omega_{u k}^{i}, \Gamma_{u k}^{r}}=\operatorname{diag}\left\{-F_{j, s}, \ldots,-F_{j, s}\right\}, \\
& \widetilde{\Psi}=\left[\begin{array}{lll}
\Psi & 0 & 0
\end{array}\right], \\
& \Psi=\left[\begin{array}{lllll}
\bar{A}-B_{1} K I_{1} & B_{2} K I_{1} & -I_{2} L \bar{C} & I
\end{array}\right], \\
& \Upsilon=\bar{A}-B_{1} K I_{1}-I .
\end{aligned}
$$

Proof. Equation (11) is equivalent to

$$
\Lambda+\widetilde{\Psi}^{T} \sum_{j=\tau_{m}}^{\tau_{M}} \sum_{s=d_{m}}^{d_{M}} \lambda_{i j} \pi_{r s} P_{j, s} \widetilde{\Psi}<0,
$$

that is

$$
\begin{aligned}
& \left(\sum_{j \in \Omega_{k}^{i}} \sum_{s \in \Gamma_{k}^{r}} \lambda_{i j} \pi_{r s}+\sum_{j \in \Omega_{u k}^{i}} \sum_{s \in \Gamma_{k}^{r}} \lambda_{i j} \pi_{r s}+\sum_{j \in \Omega_{k}^{i}} \sum_{s \in \Gamma_{u k}^{r}} \lambda_{i j} \pi_{r s}\right. \\
& \left.+\sum_{j \in \Omega_{u k}^{i}} \sum_{s \in \Gamma_{u k}^{r}} \lambda_{i j} \pi_{r s}\right) \Lambda+\widetilde{\Psi}^{T}\left(\sum_{j \in \Omega_{k}^{i}} \sum_{s \in \Gamma_{k}^{r}} \lambda_{i j} \pi_{r s} P_{j, s}\right. \\
& +\sum_{j \in \Omega_{u k}^{i}} \sum_{s \in \Gamma_{k}^{r}} \lambda_{i j} \pi_{r s} P_{j, s}+\sum_{j \in \Omega_{k}^{i}} \sum_{s \in \Gamma_{u k}^{r}} \lambda_{i j} \pi_{r s} P_{j, s} \\
& \left.+\sum_{j \in \Omega_{u k}^{i}} \sum_{s \in \Gamma_{u k}^{r}} \lambda_{i j} \pi_{r s} P_{j, s}\right) \Psi<0 .
\end{aligned}
$$

The above inequality can be written as

$$
\begin{aligned}
\sum_{j \in \Omega_{k}^{i}} \sum_{s \in \Gamma_{k}^{r}} \lambda_{i j} \pi_{r s} \Lambda+\widetilde{\Psi}^{T} \sum_{j \in \Omega_{k}^{i}} \sum_{s \in \Gamma_{k}^{r}} \lambda_{i j} \pi_{r s} P_{j, s} \Psi \\
+\sum_{j \in \Omega_{u k}^{i}} \lambda_{i j}\left(\sum_{s \in \Gamma_{k}^{r}} \pi_{r s} \Lambda+\widetilde{\Psi}^{T} \sum_{s \in \Gamma_{k}^{r}} \pi_{r s} P_{j, s} \Psi\right)
\end{aligned}
$$

$$
\begin{aligned}
& +\sum_{s \in \Gamma_{u k}^{r}} \pi_{r s}\left(\sum_{j \in \Omega_{k}^{i}} \lambda_{i j} \Lambda+\widetilde{\Psi}^{T} \sum_{j \in \Omega_{k}^{i}} \pi_{r s} P_{j, s} \Psi\right) \\
& +\sum_{j \in \Omega_{u k}^{i}} \sum_{s \in \Gamma_{u k}^{r}} \lambda_{i j} \pi_{r s}\left(\Lambda+\widetilde{\Psi}^{T} P_{j, s} \Psi\right)<0 .
\end{aligned}
$$

By Schur complement Lemma,

$$
\sum_{j \in \Omega_{k}^{i}} \sum_{s \in \Gamma_{k}^{r}} \lambda_{i j} \pi_{r s} \Lambda+\sum_{j \in \Omega_{k}^{i}} \sum_{s \in \Gamma_{k}^{r}} \lambda_{i j} \pi_{r s} \widetilde{\Psi}^{T} P_{j, s} \Psi<0,
$$

is equivalent to the following inequality

$$
\left[\begin{array}{cc}
\sum_{j \in \Omega_{k}^{i}} \sum_{s \in \Gamma_{k}^{r}} \lambda_{i j} \pi_{r s} \Lambda & * \\
\Theta_{\Omega_{k}^{i}, r_{k}^{r}} & -\widetilde{\Delta}_{\Omega_{k}^{i}, r_{k}^{r}}
\end{array}\right]<0,
$$

where $\widetilde{\Delta}_{\Omega_{k}^{i} \Gamma_{k}^{r}}=\operatorname{diag}\left\{-P^{-1} \Omega_{k_{1}^{i} \Gamma_{k_{1}^{r}}^{r}}, \ldots,-P^{-1} \Omega_{k_{\mu}^{i} \Gamma_{\theta}^{k}}\right\}$. Let $P_{j, s}^{-1}=$ $F_{j, s}, j \in \Omega, s \in \Gamma$, and $Z_{l}^{-1}=Y_{l}, l \in\{1,2,3,4\}$; we can obtain (33) and (37). Hence, (42) holds if (33) holds. Because $\lambda_{i j} \geq 0$, $\pi_{r s} \geq 0$, it can be concluded that if (33)-(36) holds, (39) holds, which completes the proof.

The conditions in Theorem 7 are in fact a set of linear matrix inequalities (LMIs) with some inversion constraints. Though they are nonconvex which brings difficulties in 
solving them using the existing convex optimization tool, we can use the cone complementarity linearization (CCL) algorithm [22] to transform this problem into the nonlinear minimization problem with LMIs constraints as follows:

$$
\begin{aligned}
& \min \operatorname{tr}\left(\sum_{l=1}^{4} Z_{l} Y_{l}+\sum_{j=\tau_{m}}^{\tau_{M}} \sum_{s=d_{m}}^{d_{M}} P_{j, s} F_{j, s}\right) \\
& \text { s.t. } \quad(27),(45), \text { and }(46) \\
& {\left[\begin{array}{cc}
Z_{l} & I \\
I & Y_{l}
\end{array}\right]>0, \quad l \in\{1,2,3,4\},} \\
& {\left[\begin{array}{cc}
P_{j, s} & I \\
I & F_{j, s}
\end{array}\right]>0, \quad j \in \Omega, s \in \Gamma .}
\end{aligned}
$$

Furthermore, the iterative algorithm which can be used to calculate the controller gain matrix $K$ and observer gain matrix $L$ is given as follows.

\section{Algorithm 9.}

Step 1. Find a feasible solution satisfying (27), (45), and (46) and set as $\left(Z_{1}^{0}, Z_{2}^{0}, Z_{3}^{0}, Z_{4}^{0}, Y_{1}^{0}, Y_{2}^{0}, Y_{3}^{0}, Y_{4}^{0}, P_{j, s}^{0}, F_{j, s}^{0}, K^{0}, L^{0}\right)$; Let $k=0$.

Step 2. Solve the following LMI optimization problem for variables $\left(Z_{1}, Z_{2}, Z_{3}, Z_{4}, Y_{1}, Y_{2}, Y_{3}, Y_{4}, P_{j, s}, F_{j, s}, K, L\right)$. $\min \operatorname{tr}\left(\sum_{l=1}^{4}\left(Z_{l}^{k} Y_{l}+R_{l} Y_{l}^{k}\right)+\sum_{j=\tau_{m}}^{\tau_{M}} \sum_{s=d_{m}}^{d_{M}}\left(P_{j, s}^{k} F_{j, s}+P_{j, s} F_{j, s}^{k}\right)\right)$, subject to (27), (45), and (46); set $\left(Z_{1}^{k}=Z_{1}, Y_{1}^{k}=Y_{1}\right.$, $Z_{2}^{k}=Z_{2}, Y_{2}^{k}=Y_{2}, Z_{3}^{k}=Z_{3}, Y_{3}^{k}=Y_{3}, Z_{4}^{k}=Z_{4}, Y_{4}^{k}=Y_{4}$, $\left.P_{j, s}^{k}=P_{j, s}, F_{j, s}^{k}=F_{j, s}, K^{k}=K, L^{k}=L\right)$.

Step 3. If (27) and (28) are satisfied, then exit the iteration. Otherwise, let $k=k+1$, and return to Step 2 .

The conditions in Theorem 8 can be solved by the similar procedures.

Remark 10. Making use of Matlab LMI tool box, a feasible solution satisfying (27), (45), and (46) can be obtained by the functions feasp() and $\operatorname{dec} 2 \mathrm{mat}()$. Once the feasible solution $\left(Z_{1}^{0}, Z_{2}^{0}, Z_{3}^{0}, Z_{4}^{0}, Y_{1}^{0}, Y_{2}^{0}, Y_{3}^{0}, Y_{4}^{0}, P_{j, s}^{0}, F_{j, s}^{0}, K^{0}, L^{0}\right)$ is obtained, then the LMI optimization problem $\min \operatorname{tr}\left(\sum_{l=1}^{4}\left(Z_{l}^{k} Y_{l}+\right.\right.$ $\left.\left.Z_{l} Y_{l}^{k}\right)+\sum_{j=\tau_{m}}^{\tau_{M}} \sum_{s=d_{m}}^{d_{M}}\left(P_{j, s}^{k} F_{j, s}+P_{j, s} F_{j, s}^{k}\right)\right)$, subject to (27), (45), and (46), can be solved by the functions $\operatorname{defcx}()$ and $\operatorname{mincx}()$.

\section{Numerical Example}

In this section, we present two examples to illustrate the effectiveness of the proposed method.

Example 1. Consider the nonlinear controlled plant with the following parameter:

$$
A=\left[\begin{array}{cc}
0.52 & -0.69 \\
0 & 0.19
\end{array}\right] \text {, }
$$

$$
\begin{aligned}
B & =\left[\begin{array}{l}
0.3 \\
0.2
\end{array}\right], \\
C & =\left[\begin{array}{ll}
1.5 & 0.7 \\
0.2 & 0.3
\end{array}\right], \\
g & =0.1 \\
f\left(k, x_{k}\right) & =0.1 \sin x_{k} .
\end{aligned}
$$

We can see that the linear part of the control plant is stable itself. The random S-C delay $\tau_{k} \in\{0,1\}$ and C-A delay $d_{k} \in\{0,1\}$. Their transition probability matrices are as follows, respectively:

$$
\begin{aligned}
& \Xi=\left[\begin{array}{cc}
0.7 & 0.3 \\
? & ?
\end{array}\right], \\
& \Pi=\left[\begin{array}{cc}
? & ? \\
0.2 & 0.8
\end{array}\right] .
\end{aligned}
$$

By Theorem 7, we can obtain the controller gain matrix $K$ and observer gain matrix $L$ as follows:

$$
\begin{aligned}
K & =\left[\begin{array}{ll}
-0.0424 & 0.1725
\end{array}\right], \\
L & =\left[\begin{array}{ll}
0.5519 & -2.6383 \\
0.1065 & -0.6730
\end{array}\right] .
\end{aligned}
$$

The initial value $x_{0}=\left[\begin{array}{ll}-1 & 1\end{array}\right]^{T}, \widehat{x}_{0}=\left[\begin{array}{ll}-0.9 & -1.1\end{array}\right]^{T}, \tau_{0}=d_{0}=$ 0 . The trajectories of the closed-loop system's states and the corresponding estimated value are shown in Figures 2 and 3 which indicate that the closed-loop system is stochastically stable.

By Theorem 8, the controller gain matrix $K$ and observer gain matrix $L$ can also be obtained as follows:

$$
\begin{aligned}
K & =\left[\begin{array}{ll}
-0.0802 & 0.2425
\end{array}\right], \\
L & =\left[\begin{array}{ll}
0.2968 & -1.7036 \\
0.1569 & -1.0017
\end{array}\right]
\end{aligned}
$$

The trajectories of the closed-loop system's states and the corresponding estimated value are omitted.

Example 2. Consider the classical angular positioning system shown in Figure 4, which consists of a rotating antenna at the origin of the plane driven by a motor [23].

Assume that the angular position of the antenna $\varphi$, the angular position of the moving object $\varphi_{r}$, and the angular velocity of the antenna $\dot{\varphi}$ are measurable. The state variables 


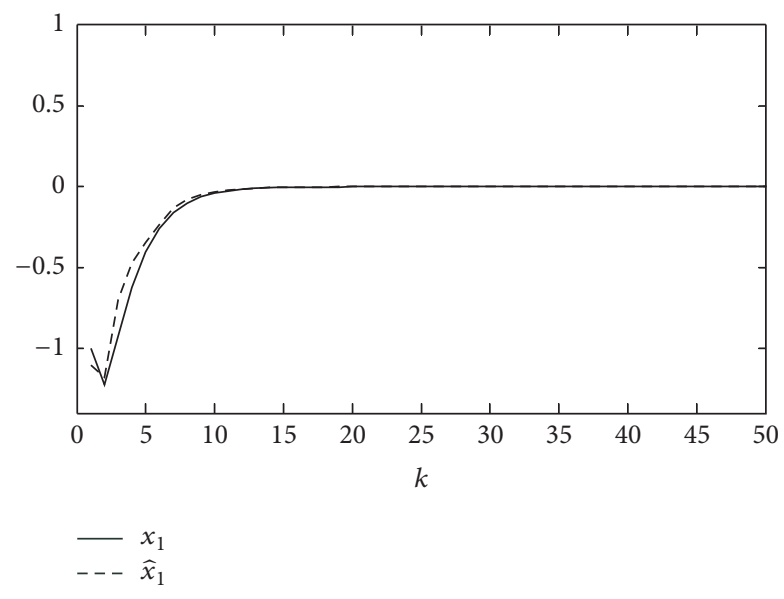

FIgURE 2: State $x_{1}$ and its estimated value $\widehat{x}_{1}$.

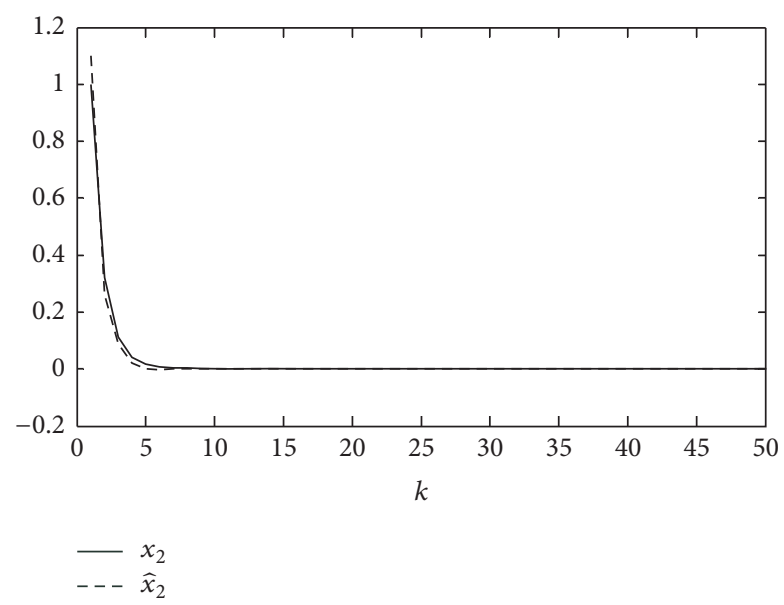

FIGURE 3: State $x_{2}$ and its estimated value $\widehat{x}_{2}$.

are chosen as $\left[\begin{array}{ll}\varphi & \dot{\varphi}\end{array}\right]^{T}$; then state equation of the angular positioning system can be obtained with the following parameter, where $f\left(k, x_{k}\right)$ can be treated as the external disturbance:

$$
\begin{aligned}
A & =\left[\begin{array}{ll}
1 & 0.0995 \\
0 & 0.99
\end{array}\right], \\
B & =\left[\begin{array}{l}
0.0039 \\
0.0783
\end{array}\right], \\
C & =\left[\begin{array}{ll}
1.4 & 0.8 \\
-0.2 & 0.4
\end{array}\right], \\
g & =0.2, \\
f\left(k, x_{k}\right) & =\frac{0.2 \sin x_{k}}{k} .
\end{aligned}
$$

Obviously, it can be seen that the linear part of the control plant is unstable. The random S-C delay $\tau_{k} \in\{0,1\}$ and C-A

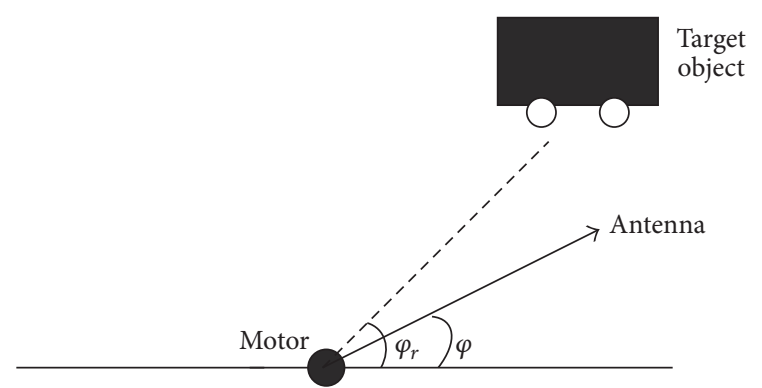

FIGURE 4: The angular positioning system.

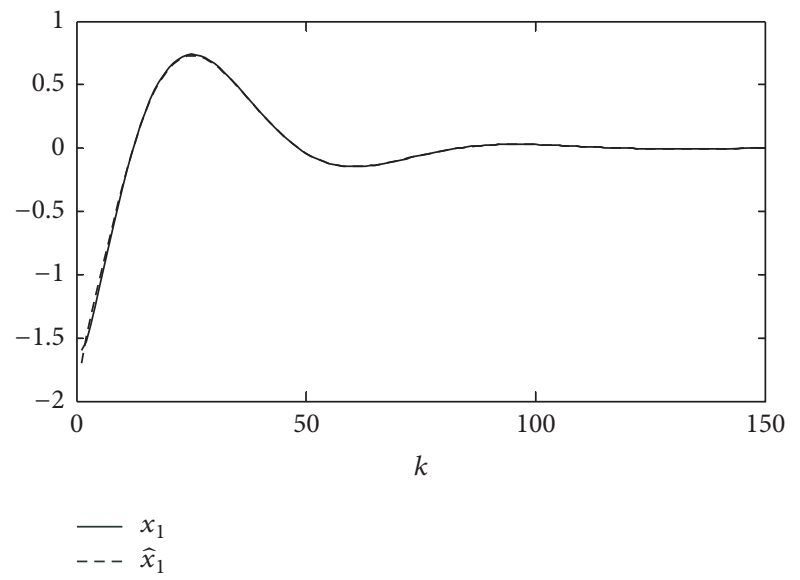

FIgURE 5: State $x_{1}$ and its estimated value $\widehat{x}_{1}$.

delay $d_{k} \in\{0,1\}$. Their transition probability matrices are as follows, respectively:

$$
\begin{aligned}
& \Xi=\left[\begin{array}{cc}
? & ? \\
0.6 & 0.4
\end{array}\right], \\
& \Pi=\left[\begin{array}{cc}
0.2 & 0.8 \\
? & ?
\end{array}\right] .
\end{aligned}
$$

For this unstable plant, we cannot get the controller gain matrix $K$ and observer gain matrix $L$ by Theorem 7 due to its conservativeness. However, by Theorem 8 , we can obtain $K$ and $L$ as follows:

$$
\begin{aligned}
K & =\left[\begin{array}{ll}
-1.1957 & -1.1143
\end{array}\right], \\
L & =\left[\begin{array}{cc}
0.1943 & -0.2715 \\
0.1130 & 0.6251
\end{array}\right] .
\end{aligned}
$$

The initial value $x_{0}=\left[\begin{array}{ll}-1.6 & 1.7\end{array}\right]^{T}, \widehat{x}_{0}=\left[\begin{array}{ll}-1.7 & 1.6\end{array}\right]^{T}, \tau_{0}=$ $d_{0}=0$. The trajectories of the closed-loop system's states and the corresponding estimated value are shown in Figures 5 and 6 which indicate that the closed-loop system is stochastically stable.

Remark 11 . The results in this paper only require a part of transition probabilities while the results in $[13,14]$ need the full information of the transition probabilities. In view of 


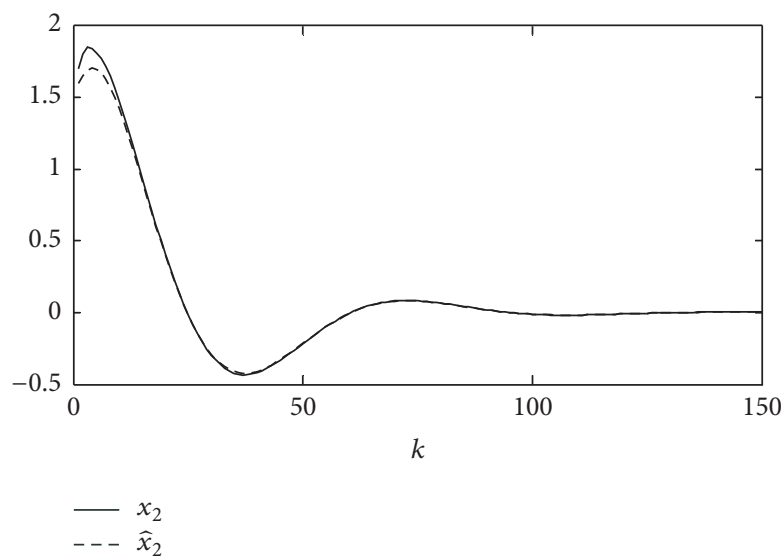

Figure 6: State $x_{2}$ and its estimated value $\widehat{x}_{2}$.

these, the proposed controller design method is more general than that of $[13,14]$.

\section{Conclusion}

The observer-based controller design problem for a class of nonlinear networked control systems with random timedelays is investigated in this paper. Two dependent Markov chains are employed to describe S-C delay and C-A delay, respectively. The transition probabilities of S-C delay and $\mathrm{C}-$ A delay are both assumed to be partly accessible. Sufficient conditions on the stochastic stability for the closed-loop systems are obtained by the Lyapunov stability theory. The CCL algorithm is employed to calculate the controller and observer gain matrices. Finally, two examples are used to illustrate the effectiveness of the proposed method.

\section{Conflicts of Interest}

The authors declare that they have no conflicts of interest.

\section{Acknowledgments}

The research is supported by the National Natural Science Foundation of China (Grant nos. 61503136, 61573137, 61573136, and 61603133).

\section{References}

[1] P. V. Zhivoglyadov and R. H. Middleton, "Networked control design for linear systems," Automatica, vol. 39, no. 4, pp. 743$750,2003$.

[2] D. Yue, Q. L. Han, and C. Peng, "State feedback controller design of networked control systems," IEEE Transactions on Circuits and Systems II: Express Briefs, vol. 51, no. 11, pp. 640-644, 2004.

[3] Q. Zhou, C. Wu, and P. Shi, "Observer-based adaptive fuzzy tracking control of nonlinear systems with time delay and input saturation," Fuzzy Sets and Systems, 2016.

[4] X. Tang and B. Ding, "Model predictive control of linear systems over networks with data quantizations and packet losses," Automatica, vol. 49, no. 5, pp. 1333-1339, 2013.
[5] Y. Zhang and H. Fang, "Stabilization of nonlinear networked systems with sensor random packet dropout and time-varying delay," Applied Mathematical Modelling. Simulation and Computation for Engineering and Environmental Systems, vol. 35, no. 5, pp. 2253-2264, 2011.

[6] E. Tian, D. Yue, and X. Zhao, "Quantised control design for networked control systems," IET Control Theory and Applications, vol. 1, no. 6, pp. 1693-1699, 2007.

[7] J. Nilsson, Real-time control systems with delays [Ph.D. thesis], Lund Institute of Technology, Lund, Sweden, 1998.

[8] L. Xiao, A. Hassibi, and J. P. How, "Control with random communication delays via a discrete-time jump system approach," in Proceedings of the American Control Conference, pp. 21992204, IEEE, Chicago, Ill, USA, June 2000.

[9] S. W. Gao and G. Y. Tang, "Output feedback stabilization of networked control systems with random delays," in Proceedings of the 18th IFAC World Congress, pp. 3250-3255, Milan, Italy, 2011.

[10] P. Shi and E. K. Boukas, " $H_{\infty}$ control for markovian jumping linear systems with parametric uncertainty," Journal of Optimization Theory and Applications, vol. 95, no. 1, pp. 75-99, 1997.

[11] P. Shi, E.-K. Boukas, and R. K. Agarwal, "Control of Markovian jump discrete-time systems with norm bounded uncertainty and unknown delay," Institute of Electrical and Electronics Engineers. Transactions on Automatic Control, vol. 44, no. 11, pp. 2139-2144, 1999.

[12] O. L. V. Costa and R. P. Marques, "Robust $\mathrm{H}_{2}$ control for discrete-time Markovian jump linear systems," International Journal of Control, vol. 73, no. 1, pp. 11-21, 2000.

[13] L. Q. Zhang, Y. Shi, T. W. Chen, and B. Huang, "A new method for stabilization of networked control systems with random delays," IEEE Transactions on Automatic Control, vol. 50, no. 8, pp. 1177-1181, 2005.

[14] Y. Shi and B. Yu, "Output feedback stabilization of networked control systems with random delays modeled by Markov chains," IEEE Transactions on Automatic Control, vol. 54, no. 7, pp. 1668-1674, 2009.

[15] Q. Li, B. G. Xu, and S. B. Li, "H-infinity control for networked control systems with data packet dropouts and partly unknown transition probabilities," Control Theory \& Applications, vol. 28, no. 8, pp. 1105-1112, 2011.

[16] S. Q. Yu and J. M. Li, "Robust stabilization of networked control systems with nonlinear perturbation," Control and Decision, vol. 27, no. 12, pp. 1917-1920, 2012.

[17] Y. F. Wang, P. L. Wang, H. Y. Chen, and M. Fang, "State feedback for networked control systems with partly unknown transition probabilities," Control Engineering of China, vol. 22, no. 4, pp. 776-779, 2015.

[18] Y. F. Wang, P. L. Wang, Z. X. Li, and H. Y. Chen, "Fault-tolerant control for networked control systems with limited information in case of actuator fault," Mathematical Problems in Engineering, vol. 2015, Article ID 785289, 7 pages, 2015.

[19] X. F. Jiang, Q.-L. Han, and X. H. Yu, "Stability criteria for linear discrete-time systems with interval-like time-varying delay," in Proceedings of the American Control Conference (ACC '05), pp. 2817-2822, IEEE, Portland, Ore, USA, June 2005.

[20] J. G. Li, J. Q. Yuan, and J. G. Lu, “Observer-based $\mathrm{H}_{\infty}$ control for networked nonlinear systems with random packet losses," ISA Transactions, vol. 49, no. 1, pp. 39-46, 2010.

[21] Y. F. Wang, P. L. Wang, and Z. D. Cai, "Robust H-infinity fault detection for networked control systems with partly 
unknown time-delay transition probabilities," Control Theory \& Applications, vol. 34, no. 2, pp. 273-279, 2017.

[22] L. Zhang, B. Huang, and J. Lam, " $H_{\infty}$ model reduction of Markovian jump linear systems," Systems \& Control Letters, vol. 50, no. 2, pp. 103-118, 2003.

[23] L. Qiu, F. Yao, and X. Zhong, "Stability analysis of networked control systems with random time delays and packet dropouts modeled by Markov chains," Journal of Applied Mathematics, vol. 2013, Article ID 715072, 10 pages, 2013. 


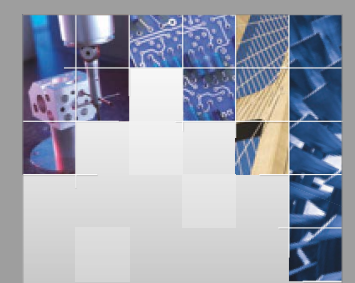

\section{Enfincering}
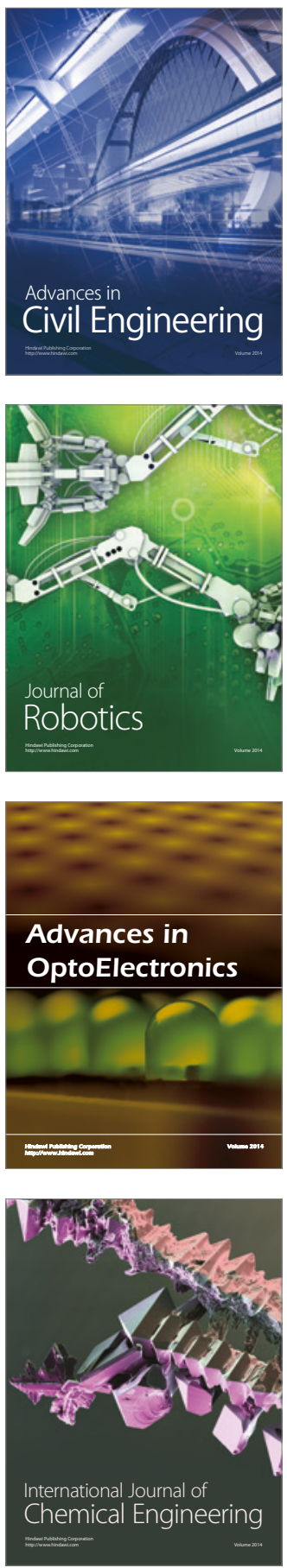

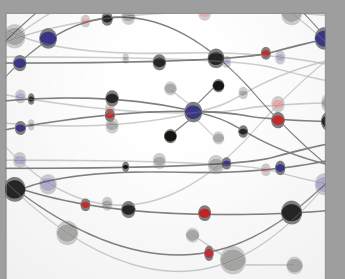

The Scientific World Journal

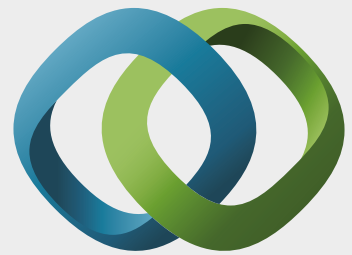

\section{Hindawi}

Submit your manuscripts at

https://www.hindawi.com
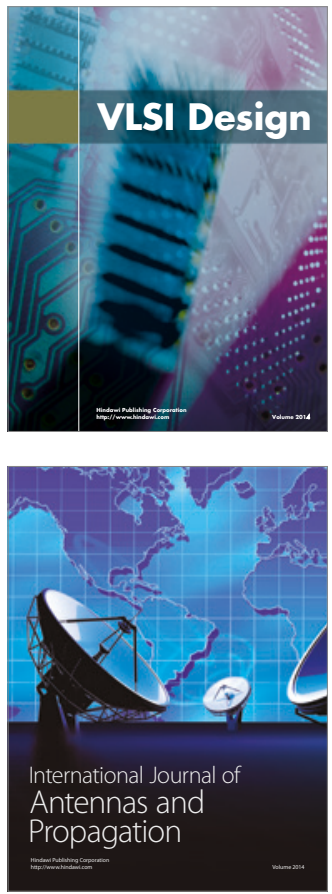

\section{Rotating}

Machinery
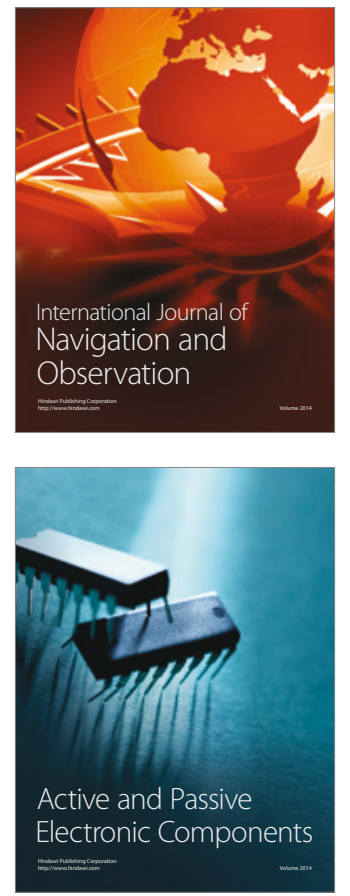
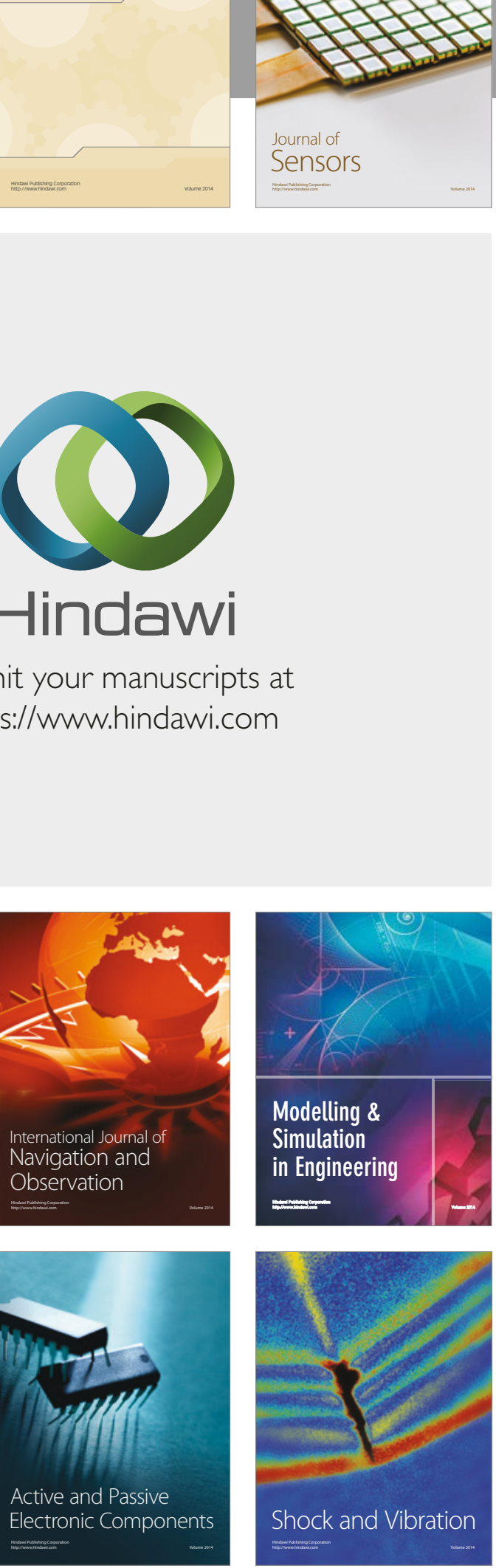
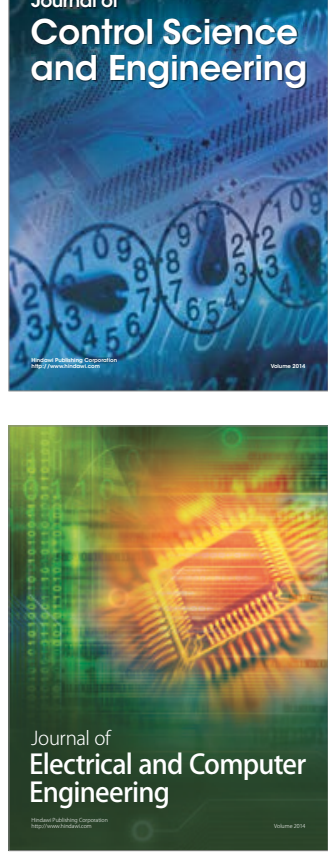

Distributed

Journal of

Control Science

and Engineering
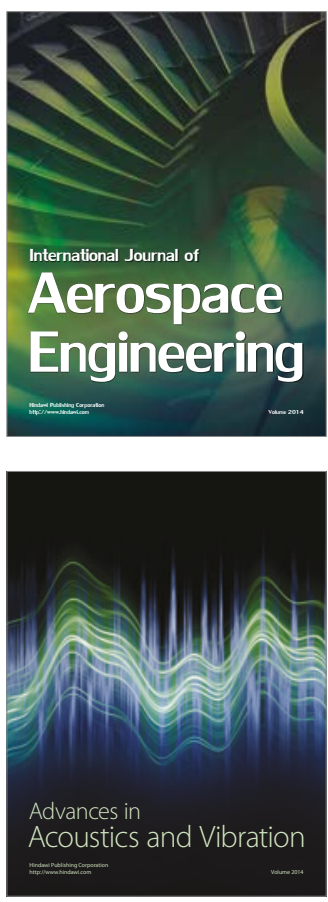

Sensor Networks 